\title{
Outcomes After Minimally-invasive Versus Open Pancreatoduodenectomy
}

\author{
A Pan-European Propensity Score Matched Study
}

\author{
Sjors Klompmaker, MD, ${ }^{*}$ Jony van Hilst, MD, MSc, ${ }^{*}$ Ulrich F. Wellner, MD, $\dagger$ \\ Olivier R. Busch, MD, PhD, ${ }^{*}$ Andrea Coratti, MD, § Mathieu D’Hondt, MD, $\dagger$ Safi Dokmak, MD, $\ddagger$ \\ Sebastiaan Festen, MD, PhD, ${ }^{* * *}$ Mustafa Kerem, MD, †† Igor Khatkov, MD, PhD, ||||

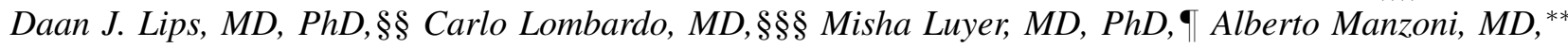 \\ Izaäk Q. Molenaar, MD, PhD,**** Edoardo Rosso, MD, ,* Olivier Saint-Marc, MD, $\uparrow \uparrow$ \\ Franky Vansteenkiste, MD, $\dagger$ Uwe A. Wittel, MD, $\ddagger \dagger \dagger$ Bert Bonsing, MD, PhD, $\uparrow$ \\ Bas Groot Koerkamp, MD, PhD, || Mohammed Abu Hilal, MD, PhD, FRCS, FACS, ||||| \\ David Fuks, MD, PhD, 㧊 Ignasi Poves, MD, PhD, \\ Ugo Boggi, MD, $\S \S$ and Marc G. Besselink, MD, MSc, PhD*, for the \\ European consortium on Minimally Invasive Pancreatic Surgery (E-MIPS)
}

Objective: To assess short-term outcomes after minimally invasive (laparoscopic, robot-assisted, and hybrid) pancreatoduodenectomy (MIPD) versus open pancreatoduodenectomy (OPD) among European centers.

Background: Current evidence on MIPD is based on national registries or single expert centers. International, matched studies comparing outcomes for MIPD and OPD are lacking.

From the *Department of Surgery, Cancer Center Amsterdam, Academic Medical Center, University of Amsterdam, the Netherlands; $†$ Department of Digestive and Hepatobiliary/Pancreatic Surgery, Groeninge Hospital, Kortrijk, Belgium; tDepartment of HPB Surgery and Liver Transplantation, Beaujon Hospital, Clichy, France; §Department of Oncology and Robotic Surgery, Careggi University Hospital, Florence, Italy; -Department of Surgery, Catharina Hospital Eindhoven, Eindhoven, the Netherlands; |Department of Surgery, Erasmus MC, Rotterdam, the Netherlands; **Department of Surgery, Fondazione Poliambulanza - Istituto Ospedaliero, Brescia, Italy; ††Department of General Surgery, School of Medicine, Gazi University, Ankara, Turkey; $\ddagger \ddagger D e p a r t m e n t$ of Surgery, Hospital del Mar, Barcelona, Spain; $\S \S D e p a r t m e n t$ of Surgery, Jeroen Bosch Hospital, s-Hertogenbosch, the Netherlands; $\uparrow$ 'Department of Surgery, Leiden University Medical Center, Leiden, the Netherlands; ||||Department of Surgery, Moscow Clinical Scientific Center, Moscow, Russia; ${ }^{* * *}$ Department of Surgery, OLVG, Amsterdam, the Netherlands; †† Department of Surgery, UKSH Campus Lübeck, Lübeck, Germany; ItłDepartment of Visceral and General Surgery, University of Freiburg Medical Center, Freiburg, Germany; §§§Division of General and Transplant Surgery, University of Pisa, Pisa, Italy; $\uparrow \uparrow \uparrow$ Department of Surgery, Center Hospitalier Régional Orleans, Orleans, France; ||||||Department of Surgery, Southampton University Hospital NHS Foundation Trust, Southampton, United Kingdom; $* * * *$ Department of Surgery, University Medical Center Utrecht, Utrecht, the Netherlands; $\dagger \dagger \dagger \dagger$ Deutsche Gesellschaft für Allgemein- und Viszeralchirurgie (DGAV), Studien- Dokumentations- und

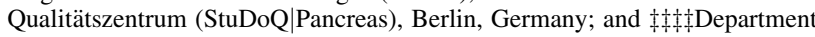
of Digestive, Oncological and Metabolic Surgery, Institut Mutualiste Montsouris, Université Paris Descartes, Paris, France.

Supplemental digital content is available for this article. Direct URL citations appear in the printed text and are provided in the HTML and PDF versions of this article on the journal's Web site (www.annalsofsurgery.com).

Disclosure: This study was registered at clinicaltrials.gov (NCT03172572). MAH, DF, IP, TK, UB, and MGB are principal investigators and shared senior authors. The authors declare no conflict of interests.

Reprints: Marc G. Besselink, MD, MSc, PhD, Sjors Klompmaker, MD, Cancer Center Amsterdam, Academic Medical Center, University of Amsterdam, Meibergdreef 9, 1105 AZ Amsterdam, the Netherlands. E-mails: m.g.besselink@amc.nl, s.klompmaker@amc.nl.

Copyright (C) 2019 Wolters Kluwer Health, Inc. All rights reserved.

ISSN: 0003-4932/19/27102-0356

DOI: $10.1097 /$ SLA.0000000000002850
Methods: Retrospective propensity score matched study comparing MIPD in 14 centers ( 7 countries) performing $\geq 10$ MIPDs annually (2012-2017) versus OPD in 53 German/Dutch surgical registry centers performing $\geq 10$ OPDs annually (2014-2017). Primary outcome was 30-day major morbidity (Clavien-Dindo $\geq 3$ ).

Results: Of 4220 patients, 729/730 MIPDs (412 laparoscopic, 184 robotassisted, and 130 hybrid) were matched to 729 OPDs. Median annual casevolume was 19 MIPDs (interquartile range, IQR 13-22), including the first MIPDs performed in 10/14 centers, and 31 OPDs (IQR 21-38). Major morbidity $(28 \%$ vs $30 \%, P=0.526)$, mortality $(4.0 \%$ vs $3.3 \%, P=$ $0.576)$, percutaneous drainage ( $12 \%$ vs $12 \%, P=0.809)$, reoperation (11\% vs $13 \%, P=0.329$ ), and hospital stay (mean 17 vs 17 days, $P>$ $0.99)$ were comparable between MIPD and OPD. Grade-B/C postoperative pancreatic fistula (POPF) $(23 \%$ vs $13 \%, P<0.001)$ occurred more frequently after MIPD. Single-row pancreatojejunostomy was associated with POPF in MIPD (odds ratio, OR 2.95, $P<0.001$ ), but not in OPD. Laparoscopic, robotassisted, and hybrid MIPD had comparable major morbidity (27\% vs $27 \%$ vs $35 \%$ ), POPF (24\% vs $19 \%$ vs $25 \%$ ), and mortality ( $2.9 \%$ vs $5.2 \%$ vs $5.4 \%$ ), with a fewer conversions in robot-assisted- versus laparoscopic MIPD (5\% vs $26 \%, P<0.001)$.

Conclusions: In the early experience of 14 European centers performing $\geq 10$ MIPDs annually, no differences were found in major morbidity, mortality, and hospital stay between MIPD and OPD. The high rates of POPF and conversion, and the lack of superior outcomes (ie, hospital stay, morbidity) could indicate that more experience and higher annual MIPD volumes are needed.

Keywords: hybrid, laparoscopic, minimally invasive, pancreas, pancreatic cancer, pancreatic tumors, propensity score matching, robot, robotic, surgery, Whipple

(Ann Surg 2020;271:356-363)

D ancreatoduodenectomy is associated with high morbidity rates and a strong volume-outcome relationship. ${ }^{1-3}$ Minimally invasive pancreatoduodenectomy (MIPD; laparoscopic, robot-assisted, or hybrid) could reduce morbidity and enhance postoperative recovery compared with open pancreatoduodenectomy (OPD), ${ }^{1,2,4-12}$ but its implementation is lagging compared with minimally invasive distal pancreatectomy. ${ }^{13,14}$

Two factors may have delayed the implementation of MIPD. First, pancreatoduodenectomy is a demanding procedure with several anastomoses and potentially life-threatening complications. Indeed, 
MIPD has been associated with increased perioperative mortality compared with OPD in centers performing a fewer than 10 MIPDs annually. ${ }^{15,16}$ Moreover, improved outcomes in centers performing more than 40 OPDs annually could indicate that the optimal volume cut-off for MIPD is also (much) higher. ${ }^{17}$ Second, current evidence on the effectiveness and safety of MIPD consists mainly of registry studies with a large proportion of low-volume centers ${ }^{14-16,18}$ or small-/single center retrospective studies from high-volume centers, suffering from reporting bias and/or insufficient adjustment for confounding by indication (surgical case selection). ${ }^{1,8}$ The only published randomized trial on laparoscopic MIPD versus OPD (PLOT: Pancreatic Head and Periampullary Cancer Laparoscopic versus Open Surgical Treatment; NCT02081131) reported shorter length of hospital stay after MIPD but was underpowered to demonstrate a benefit regarding major morbidity. ${ }^{19}$ Large multicenter (matched) studies on MIPD versus OPD are lacking.

The purpose of this study was to combine data from European centers performing at least 10 MIPDs annually in a multicenter propensity score matched cohort study on MIPD versus OPD. We hypothesized that MIPD is associated with equivalent morbidity and mortality compared with OPD, when performed in such centers, but with superior secondary outcomes (eg, shorter length of stay).

\section{METHODS}

We performed a retrospective multicenter propensity score matched cohort study comparing MIPD cases to OPD controls. MIPD patients were included from European centers performing at least 10 MIPDs per year. OPD patients were included from centers performing at least 10 OPDs per year in 2 Dutch and German surgical registries. This study was initiated by the European Consortium on Minimally Invasive Pancreatic Surgery (E-MIPS) and supported by the Scientific and Research Committee of the European-African Hepato-Pancreato-Biliary Association (E-AHPBA). We used the STROBE guideline ${ }^{20}$ for design and reporting of the study, which included registration of a study protocol at clinicaltrials.gov (NCT03172572). Need for ethical approval was waived by the institutional review board at the Academic Medical Center in Amsterdam.

\section{Eligibility and Data Collection}

Inclusion criteria were elective MIPD (including laparoscopic, robot-assisted, or hybrid procedures) or OPD in adults, for solid premalignant tumors or cysts. Hybrid was defined as laparoscopic resection and open reconstruction via (limited) laparotomy. Exclusion criteria were chronic pancreatitis as indication for surgery, arterial resection, insufficient baseline data, or missing primary outcome data.

Included cases were consecutive patients undergoing elective MIPD between January 1, 2012 and July 31, 2017 at participating EAHPBA and E-MIPS centers. Each center appointed a local study coordinator responsible for data collection and communication with the central study coordinators (SK and JH). All data was collected via an International Conference on Harmonization Good Clinical Practice (ICH-GCP) compliant on-line electronic case report form (eCRF) and data storage environment (CASTOR, CIWIT B.V., Amsterdam, the Netherlands). In addition, local study coordinators completed a survey (Google Survey, Mountain View, CA) with questions regarding methods of data collection, annual case volume, standard of care, and surgical case selection.

Included controls were consecutive patients undergoing OPD between January 1, 2014 and July 31, 2017 who were registered in the nationwide German Society for General and Visceral Surgery (DGAV) Studien-, Dokumentations- und Qualitätszentrum (StuDoQ $\mid$ Pancreas $),{ }^{21}$ and the Dutch Pancreatic Cancer Audit (DPCA) ${ }^{22}$ registries. All centers were blinded to maintain anonymity on outcomes and prevent reporting bias.

\section{Primary and Secondary Outcomes}

The primary outcome was 30-day major morbidity (ClavienDindo 3a-5). ${ }^{23}$ Secondary outcomes were 30 -day mortality, grade-B/ $\mathrm{C}$ pancreatic fistula (POPF), grade $\mathrm{B} / \mathrm{C}$ postpancreatectomy hemorrhage (PPH), and length of hospital stay (day of surgery to day of discharge).

\section{Definitions}

Preoperative variables included baseline characteristics, such as age, sex, body-mass-index (BMI), and comorbidities (Charlson Comorbidity Index ${ }^{24}$ ), surgical history, computed tomography/magnetic resonance imaging (CT/MRI)-scan information (vascular/ organ involvement), American Society of Anesthesiologists (ASA) classification $^{25}$, and Eastern Cooperative Oncology Group (ECOG) performance status. Conversion was recorded if a robot-assisted or laparoscopic MIPD was converted to OPD. The International Study Group on Pancreatic Surgery (ISGPS) 2005 definition ${ }^{26}$ was used to classify POPF. Although a newer definition is available, ${ }^{27}$ it was not used in the registry data and could therefore not be applied to compare outcomes. The ISGPS and International Study Group of Liver Surgery (ISGLS) definitions were used to classify delayed gastric emptying, ${ }^{28} \mathrm{PPH},{ }^{29}$ and bile leakage. ${ }^{30}$ Surgical site infections were defined using the Center for Disease Control and Prevention (CDC) definition. ${ }^{31}$ All complications (minor or major) occurring during the initial hospitalization and subsequent readmissions were recorded. Patient were followed-up until discharge or 30 days postoperatively (whichever occurred later) in the German and Dutch registries and until the last hospital visit or phone call in the MIPD centers. Therefore, all primary and secondary outcomes in this study are limited to in-hospital or 30-day events.

\section{Propensity Score Matching}

Propensity score matching was applied to achieve a balanced exposure groups at baseline (ie, minimal confounding), in accordance with the recommendations by Lonjon et al. ${ }^{32}$ The probability to undergo MIPD for each patient (ie, the propensity score) was obtained from a logistic regression model. The study entry survey was used to ensure all reported MIPD selection factors were included as covariates in the model to further reduce potential confounding by indication. Final covariates were age, sex, BMI, ASA, Charlson comorbidity index, ECOG, tumor location (pancreas vs periampullary/ distal common bile duct vs duodenum), suspected malignancy, organ involvement on imaging, multivisceral resection, porto-mesenteric vein resection, and pancreatic texture (Supplement 1, http:// links.lww.com/SLA/B435). MIPD cases were analyzed intention-totreat, regardless of conversion to open surgery, and matched in a 1:1 ratio to OPD controls based on the propensity score with a standard caliper width of 0.2 .

\section{Sensitivity Analyses}

The association between treatment group (exposure) and primary and secondary outcomes was measured using odds ratios. A first sensitivity analysis assessed the impact of volume, learning curve, hybrid surgery, conversions, and laparoscopy. This was done using multivariable-adjusted odds ratios after respective exclusion of centers performing 10 to 20 MIPDs per year, the first 20 MIPD cases at each center, hybrid procedures, hybrid and converted procedures, and hybrid and laparoscopic procedures. A second sensitivity analysis was performed to mitigate differences in postoperative t-stage, histopathological diagnosis, and pancreatic duct diameter by replacing preoperative diagnosis by histological diagnosis, and by 
including tumor stage and duct diameter in the propensity score model. A third analysis assessed baseline-, tumor-, and perioperative characteristics to identify new risk factors for POPF after MIPD, using multivariable logistic regression based on a univariable screen $(P<0.1)$ and backward stepwise elimination (if $P>0.05$ and clinically irrelevant).

\section{Statistics}

Missing baseline and outcome data were resolved using chained multiple imputation ${ }^{33}$, which reduces bias in combination with propensity score matching. ${ }^{32}$ Categorical data were reported as proportions and continuous data as mean and standard deviation (SD) or median and interquartile range (IQR) as appropriate. We used the standardized mean difference (SMD) to assess balance at baseline in both groups. Optimal balance on a parameter is generally achieved when the SMD is on or below 0.1 . To test for statistical significance (alpha 0.05), we used the Fisher exact test for categorical variables, and the student $t$-test for continuous variables (applying the central limit theorem). All confidence intervals (CI) were 95\%. All data were handled and analyzed using IBM SPSS Statistics for Windows version 23.0 (IBM Corp., Orchard Road Armonk, New York, NY), STATA version 14.1 (StataCorp LP, College Station, TX), or R's programming environment (R Foundation for Statistical Computing, Vienna, Austria).

\section{RESULTS}

We identified 903 MIPD patients from 26 E-AHPBA and E-MIPS centers and 4020 OPD patients from 70 German and Dutch centers. After exclusions, 730 MIPD patients from 14 E-MIPS centers and 3490 OPD patients from 53 high-volume DGAV and DPCA centers were included (see Fig. 1). Of all 14 MIPD centers, 7 performed laparoscopic, 4 both hybrid and laparoscopic, and 3 robotassisted MIPD. The median annual pancreatoduodenectomy (MIPD and OPD combined) volume was 41 (IQR 17-69) among the MIPD centers and 31 (IQR 21-38) among the OPD centers. The median annual MIPD volume was $19(13-22)$, which included the first MIPD case performed in 10 of $14(71 \%)$ centers. Of all included patients,

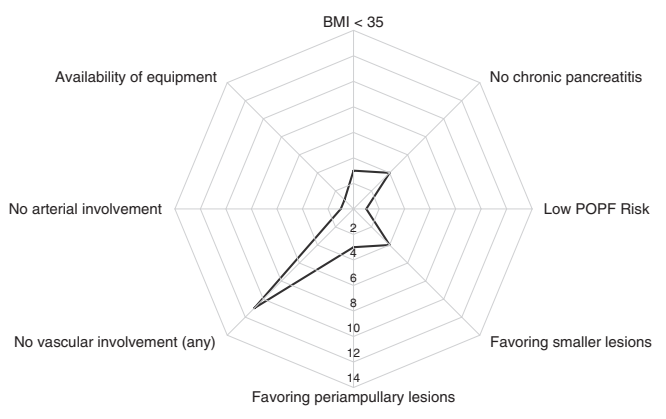

FIGURE 2. Self-reported Surgical Selection Factors for MIPD. Institutional or patient-related factors used by the 14 participating E-MIPS centers to select patients for MIPD. MIPD indicates minimally invasive pancreatoduodenectomy; POPF, postoperative pancreatic fistula.

729 of 730 MIPD cases could be matched (1:1) to an OPD control (Fig. 1).

\section{Selecting Patients for MIPD}

Reported selection factors for MIPD were absence of vascular involvement (11 of 14 MIPD centers), smaller or periampullary tumors (7 of 14), absence of chronic pancreatitis (4 of 14), BMI $\leq 35$ ( 3 of 14 ), low risk of POPF ( 1 of 14 ), absence of arterial involvement (1 of 14), and availability of minimally invasive equipment (1 of 14) (Fig. 2). One center indicated that MIPD selection factors had been stricter during the initial learning curve phase. The observed selection factors and their odds ratios are presented in Supplement 1, http://links.lww.com/SLA/B435, propensity score distributions in Supplement 2, http://links.lww.com/SLA/B435.

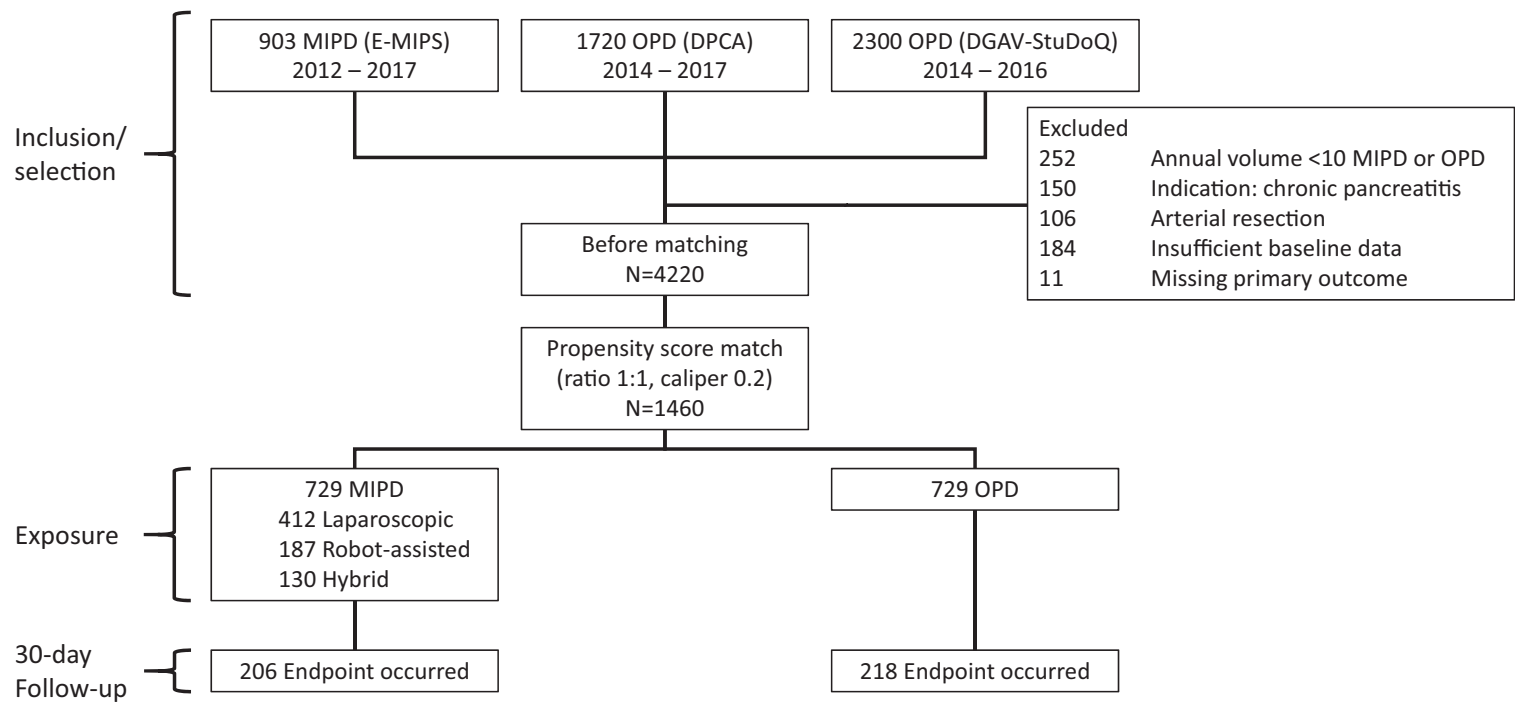

FIGURE 1. Study Flow-Chart. Endpoint was 30-day major morbidity. Annual indicates annual; DPCA, Dutch Pancreatic Cancer Audit; DGAV StuDoQ, German Society for General and Visceral Surgery Studien-, Dokumentations- und Qualitätszentrum; E-MIPS, European consortium on Minimally Invasive Pancreatic Surgery. 
TABLE 1. Baseline Characteristics Before and After Propensity Score Matching

\begin{tabular}{|c|c|c|c|c|c|}
\hline Baseline & $\begin{array}{c}\text { OPD Prematch } \\
\mathbf{N}=\mathbf{3 4 9 0}\end{array}$ & $\begin{array}{l}\text { OPD Postmatch } \\
\quad \mathbf{N}=\mathbf{7 2 9}\end{array}$ & $\begin{array}{c}\text { MIPD } \\
\mathbf{N}=\mathbf{7 2 9}\end{array}$ & $\begin{array}{c}\text { Standard } \\
\text { Difference Prematch }\end{array}$ & $\begin{array}{c}\text { Standard } \\
\text { Difference Postmatch } \\
\end{array}$ \\
\hline Age, mean (SD), y & $66.9(10.7)$ & $64.6(11.7)$ & 64.5 (11.6) & -0.21 & 0.00 \\
\hline BMI, mean (SD), $\mathrm{kg} / \mathrm{m}^{2}$ & $25.7(10.0)$ & $24.8(4.0)$ & $24.9(4.2)$ & -0.11 & 0.02 \\
\hline Charlson comorbidity index, median (IQR) & $0.6(.9)$ & $0.5(.9)$ & $0.5(.9)$ & -0.06 & -0.02 \\
\hline Female sex $(n), \%$ & $1512(43.3)$ & $363(49.8)$ & $357(49.0)$ & 0.11 & -0.02 \\
\hline \multicolumn{6}{|l|}{ ASA-classification (n), \% } \\
\hline ASA 1 & $336(9.6)$ & $154(21.1)$ & $139(19.1)$ & 0.27 & -0.06 \\
\hline ASA 2 & 1855 (53.2) & $416(57.1)$ & $421(57.8)$ & 0.09 & 0.01 \\
\hline ASA 3-4 & $1275(36.5)$ & $149(20.4)$ & $162(22.2)$ & -0.32 & 0.04 \\
\hline ASA Unknown & $24(.7)$ & $10(1.4)$ & $7(1.0)$ & & \\
\hline \multicolumn{6}{|l|}{ ECOG performance status (n), \% } \\
\hline ECOG $0-1$ & $3116(89.3)$ & $667(91.5)$ & $614(84.2)$ & -0.15 & -0.21 \\
\hline ECOG 2 & $130(3.7)$ & $31(4.3)$ & $36(4.9)$ & 0.06 & 0.03 \\
\hline ECOG 3-4 & $133(3.8)$ & $5(.7)$ & $5(.7)$ & -0.21 & 0.00 \\
\hline ECOG Unknown & $111(3.2)$ & $26(3.6)$ & $74(10.2)$ & & \\
\hline \multicolumn{6}{|l|}{ Preoperative tumor characteristics } \\
\hline \multicolumn{6}{|l|}{ Localization $(\mathrm{n}), \%$} \\
\hline Pancreas & $2208(63.3)$ & $453(62.1)$ & $458(62.8)$ & -0.01 & 0.01 \\
\hline Periampullary or CBD & 466 (13.4) & $76(10.4)$ & $101(13.9)$ & 0.01 & 0.10 \\
\hline Duodenum & $187(5.4)$ & $39(5.3)$ & $36(4.9)$ & -0.01 & -0.02 \\
\hline Unknown & $446(12.8)$ & $121(16.6)$ & $112(15.4)$ & 0.07 & -0.04 \\
\hline Malignant indication (n), \% & $2474(72.5)$ & $453(63.6)$ & $451(62.2)$ & -0.22 & -0.03 \\
\hline Neoadjuvant treatment $(\mathrm{n}), \%$ & $69(2.0)$ & $7(1.0)$ & $7(1.0)$ & -0.08 & 0.00 \\
\hline Unknown & $1(.0)$ & & $19(2.6)$ & & \\
\hline Preoperative tumor size, mean $(\mathrm{SD}), \mathrm{mm}$ & $27.5(20.3)$ & $28.9(26.8)$ & $26.4(13.9)$ & -0.06 & -0.14 \\
\hline Unknown & $2692(77.1)$ & $521(71.5)$ & $264(36.2)$ & & \\
\hline \multicolumn{6}{|l|}{ Pathology } \\
\hline \multicolumn{6}{|l|}{ Histology (n), \% } \\
\hline Adenocarcinoma - pancreas & $1690(48.4)$ & $345(47.3)$ & $307(42.1)$ & -0.13 & -0.10 \\
\hline Adenocarcinoma - duodenum & $149(4.3)$ & $23(3.2)$ & $14(1.9)$ & -0.14 & -0.07 \\
\hline Adenocarcinoma - other & $769(22.0)$ & $165(22.6)$ & $132(18.1)$ & -0.10 & -0.11 \\
\hline NET & $167(4.8)$ & $45(6.2)$ & $52(7.1)$ & 0.10 & 0.04 \\
\hline IPMN/MCN & $226(6.5)$ & $50(6.9)$ & $81(11.1)$ & 0.16 & 0.15 \\
\hline Chronic pancreatitis & $94(2.7)$ & $19(2.6)$ & $14(1.9)$ & -0.05 & -0.05 \\
\hline Intestinal adenoma & $104(3.0)$ & $23(3.2)$ & $22(3.0)$ & 0.00 & -0.01 \\
\hline Other & $269(7.7)$ & $56(7.7)$ & $100(13.7)$ & & \\
\hline Unknown & $22(.6)$ & $3(.4)$ & $7(1.0)$ & & \\
\hline \multicolumn{6}{|l|}{ T-stage (n), \% } \\
\hline Not adenocarcinoma* & $882(25.3)$ & $196(26.9)$ & $276(37.9)$ & 0.27 & 0.24 \\
\hline Tis-T2 & $424(12.1)$ & $95(13.0)$ & $142(19.5)$ & 0.20 & 0.18 \\
\hline $\mathrm{T} 3$ & $1797(51.5)$ & $355(48.7)$ & $273(37.4)$ & -0.29 & -0.23 \\
\hline $\mathrm{T} 4$ & $137(3.9)$ & $35(4.8)$ & $19(2.6)$ & -0.07 & -0.12 \\
\hline Unknown & $250(7.2)$ & $48(6.6)$ & $19(2.6)$ & & \\
\hline Tumor size, mean (SD), mm & $29.4(17.3)$ & $29.9(18.5)$ & $25.9(13.9)$ & -0.22 & -0.26 \\
\hline $\mathrm{N}$-stage $\geq 1(\mathrm{n}), \%$ & $1764(57.3)$ & $382(59.3)$ & $305(45.3)$ & -0.24 & -0.28 \\
\hline Lymph node ratio, mean (SD) & $0.1(.2)$ & $0.1(.2)$ & $0.1(.2)$ & -0.10 & -0.10 \\
\hline M-stage $\geq 1(n), \%$ & $112(3.4)$ & $19(2.8)$ & $10(1.5)$ & -0.12 & -0.09 \\
\hline
\end{tabular}

ASA indicates American Society of Anesthesiologists; BMI, body mass index; CBD, common bile duct; ECOG, Eastern Cooperative Oncology Group; IPMN, intraductal papillary mucinous neoplasm; IQR, interquartile range; MCN, mucinous cystic neoplasm; MIPD, minimally invasive pancreatoduodenectomy; NET, neuroendocrine tumor; OPD, open pancreatoduodenectomy.

*Pancreatic ductal-, duodenum-, distal bile duct-, or other type.

\section{Baseline Characteristics}

See Table 1 for baseline characteristics before and after matching. Almost all baseline variables were more balanced after propensity score matching. Some differences remained as a result of the matching by preoperative (and not postoperative) variables; the MIPD group had a fewer pathologic T-stage 3 tumors (37\% vs 49\%, SMD -0.23), smaller tumors ( $26 \mathrm{~mm}$ vs $30 \mathrm{~mm}$, SMD -0.26), and a fewer N1-tumors (45\% vs 59\%, SMD -0.28) compared with the OPD group. However, the malignant lymph node ratio ( 0.1 vs 0.1 , SMD -0.10) was similar between groups. Of note, among the 638 patients with reported pancreatic duct measurement (44\% missing), the mean duct size was slightly larger in the OPD group $(5 \mathrm{~mm}$ vs $6 \mathrm{~mm}$, SMD -0.20).

\section{Primary and Secondary Outcomes}

See Table 2 for outcomes before and after matching. The MIPD group had longer operative times [mean 416 (SD 111) vs 330 (SD 103) minutes, $P<0.001]$ and more pancreatogastrostomies $(19 \%$ vs $13 \%, P<0.002)$. Rates of routine intraoperative drain placement were similar (91\% vs $94 \%$ ) between MIPD and OPD. In the MIPD group, 115 of $729(15.8 \%)$ procedures were converted from laparoscopic $(26 \%)$ or robot-assisted $(5 \%, P<0.001)$ MIPD to OPD, see Supplement 3, http://links.lww.com/SLA/B435. The rates of major morbidity ( $28 \%$ vs $30 \%, P=0.526)$ and mortality $(4.0 \%$ vs $3.3 \%, P=0.576)$ were similar between MIPD and OPD. The rate of grade $\mathrm{B} / \mathrm{C}$ POPF $(23 \%$ vs $13 \%, P<0.001)$ was higher after MIPD. The rate of grade $\mathrm{B} / \mathrm{C}$ bile leakage was slightly lower after MIPD 
TABLE 2. Outcomes Before and After Propensity Score Matching

\begin{tabular}{|c|c|c|c|c|}
\hline Perioperative & $\begin{array}{c}\text { OPD Prematch } \\
\mathbf{N}=\mathbf{3 4 9 0}\end{array}$ & $\begin{array}{c}\text { OPD Postmatch } \\
\quad \mathbf{N}=\mathbf{7 2 9}\end{array}$ & $\begin{array}{c}\text { MIPD } \\
\mathbf{N}=\mathbf{7 2 9} \\
\end{array}$ & $\boldsymbol{P}^{* * *}$ \\
\hline Operative time, median (SD), min & $328.4(99.6)$ & $324.2(93.9)$ & $415.8(110.9)$ & $<0.001$ \\
\hline Median (IQR) & $316(259-382)$ & $312(255-377)$ & $400(330-487)$ & \\
\hline Pylorus preserving pancreatoduodenectomy & 2289 (65.6) & $501(68.7)$ & $481(66.0)$ & 0.289 \\
\hline Classic Whipple & $1201(34.4)$ & $228(31.3)$ & $240(32.9)$ & 0.537 \\
\hline Additional organs resected ${ }^{*}(\mathrm{n}), \%$ & $294(8.4)$ & $19(2.6)$ & $17(2.3)$ & 0.866 \\
\hline \multicolumn{5}{|l|}{ Type of anastomosis (n), \% } \\
\hline Pancreatojejunostomy & $2886(82.7)$ & $612(84.0)$ & $548(75.2)$ & $<0.001$ \\
\hline Pancreatogastrostomy & $456(13.1)$ & $92(12.6)$ & 136 (18.7) & 0.002 \\
\hline Other & $148(4.2)$ & $25(3.4)$ & $45(6.2)$ & \\
\hline Hard/firm pancreas & $1038(29.7)$ & $243(33.3)$ & $255(35.0)$ & 0.544 \\
\hline Unknown & $623(17.9)$ & $116(15.9)$ & 107 (14.7) & \\
\hline Pancreatic fistula grade $\mathrm{B} / \mathrm{C}(\mathrm{n}), \%$ & 469 (13.5) & $92(12.7)$ & $164(22.6)$ & $<0.001$ \\
\hline Bile leakage grade $B / C(n), \%$ & $160(4.6)$ & $37(5.1)$ & $22(3.0)$ & 0.047 \\
\hline Hemorrhage grade $B / C(n), \%$ & $274(7.9)$ & $53(7.3)$ & $69(9.5)$ & 0.156 \\
\hline Delayed gastric emptying grade $\mathrm{B} / \mathrm{C}(\mathrm{n}), \%$ & 475 (13.6) & $95(13.1)$ & $77(10.6)$ & 0.167 \\
\hline Reoperation (n), \% & 449 (13.1) & $90(12.6)$ & $80(11.0)$ & 0.329 \\
\hline Length of hospital say, mean (SD), d & $18.8(14.6)$ & $17.4(14.6)$ & $18.2(19.5)$ & $<0.001$ \\
\hline Median (IQR) & $14(11-21)$ & $13(10-19)$ & $14(9-21)$ & \\
\hline German and Dutch centers only & $18.8(14.6)$ & $17.4(14.6)$ & $17.0(12.3)$ & $>0.99$ \\
\hline Median (IQR) & $14(11-21)$ & $13(10-19)$ & $14(9-20)$ & \\
\hline Unplanned readmission (n), \% & $402(11.8)$ & $90(12.6)$ & $69(9.8)$ & 0.11 \\
\hline
\end{tabular}

${ }^{*}$ Other than pancreatic head, duodenum, gallbladder, or pylorus.

$* *$ Such as porto-mesenteric vein, superior mesenteric vein.

$* * * P$-value for the difference between OPD and MIPD after propensity-score matching.

IQR indicates interquartile range; MIPD, minimally invasive pancreatoduodenectomy; OPD, open pancreatoduodenectomy.

(3.0\% vs $5.1 \%, P=0.047)$. However, the overall rates of endoscopic reintervention $(3.7 \%$ vs $2.2 \%, P=0.068)$, percutaneous catheter drainage ( $12 \%$ vs $12 \%, P=0.809)$, reoperation $(11 \%$ vs $13 \%, P=$ $0.329)$, and intensive care unit admission $(6.3 \%$ vs $6.5 \%, P=0.500)$ were similar between MIPD and OPD.

Lengths of hospital stay [mean 18 (SD 20) vs 17 (SD 15) days and median 14 vs 13 days, $P<0.001$ ] were significantly longer in the MIPD group. As expected through variation in discharge policy between countries, there was a considerable difference in hospital stay after MIPD between 7 Belgian/Dutch/German (mean 16, median 13 days) and 5 French/Italian (mean 23, median 17 days) centers. When comparing MIPD versus OPD in Dutch and German centers, no differences in hospital stay were found [mean 17 (SD 12) vs 17 (SD 15) days - median 13 vs 14 days, $P>0.99$ ]. Equally, no significant differences were found after excluding all patients with grade B/C POPF [mean 15 (SD 11) vs 15 (SD 10) days - median 12 vs 13 days, $P=0.389]$.

\section{Sensitivity Analyses}

MIPD was not associated with major morbidity before (odds ratio, OR $0.85, P=0.072$ ) or after (OR $0.92, P=0.489)$ propensity score matching. MIPD was also not associated with 30-day mortality before (OR 1.03, $P=0.869$ ) or after (OR 1.36, $P=0.269)$, but there was an association with POPF before (OR 1.87, $P<0.001)$ and after propensity score matching (OR 2.01, $P<0.001$ ). These estimations remained stable across all sensitivity analysis scenarios (Fig. 3, Supplement 4, http://links.lww.com/SLA/B435). Between laparoscopic, robot-assisted and hybrid procedures, the unadjusted rates of major morbidity ( $27 \%$ vs $27 \%$ vs $35 \%$ ), POPF ( $24 \%$ vs $19 \%$ vs $25 \%$ ), and $30-$ day mortality ( $2.9 \%$ vs $5.2 \%$ vs $5.4 \%$ ) were similar, see Supplement 3 , http://links.lww.com/SLA/B435. Volume-outcome associations for major morbidity and POPF are presented in Supplement 5, http:// links.lww.com/SLA/B435.

In a second sensitivity analysis $(n=600)$, adding pancreatic duct diameter, histopathological diagnosis, and T-stage as propensity score matching variables, MIPD was not associated with increased 30-day major morbidity (OR $1.11, P=0.581$ ) or mortality (OR 1.61 , $P=0.253)$, and the association with POPF (OR 1.53, $P=0.087$ ) remained similar.

In a third analysis comparing MIPD patients with- $(n=164)$ and without $(\mathrm{n}=563) \mathrm{POPF}$, some known and new risk factors were identified, see Supplement 6A, http://links.lww.com/SLA/B435. After multivariable adjustment, the most important newly identified risk factor for POPF in MIPD was single-row pancreatojejunostomy (OR 2.95, $P<0.001$ ) as opposed to double-row pancreatojejunostomy or pancreatogastrostomy, see Supplement $6 \mathrm{~B}$, http://links. lww.com/SLA/B435. In patients with OPD, no association between single-row pancreatojejunostomy and POPF was found.

\section{DISCUSSION}

This large pan-European propensity score matched cohort study in centers performing at least 10 MIPDs per year found no differences in 30-day major morbidity, mortality, and length of stay between MIPD and OPD. However, MIPD was associated with a 


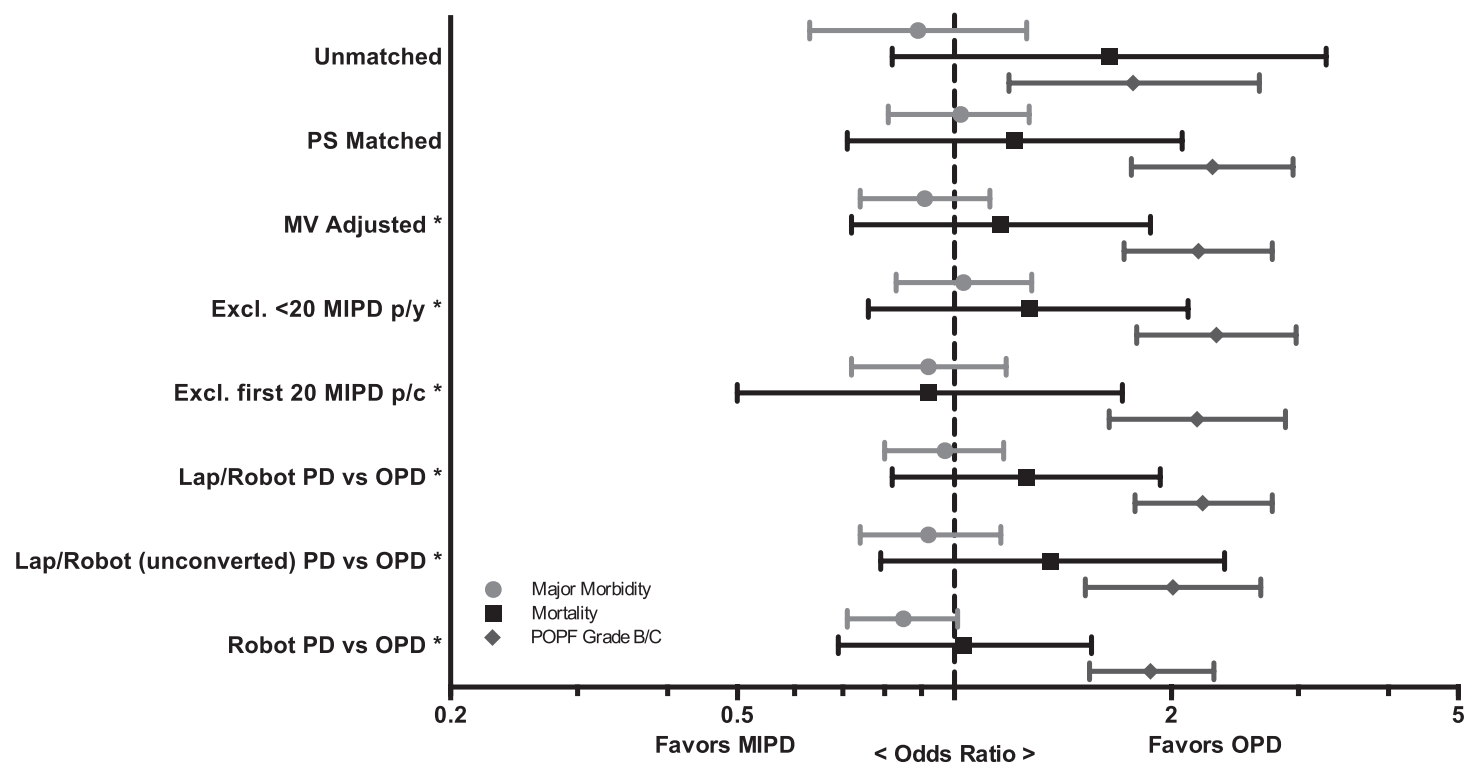

FIGURE 3. Main Outcomes and Sensitivity Analyses. Overview of the association between approach and primary (major morbidity) and secondary (mortality, postoperative pancreatic fistulae) outcomes in primary and sensitivity analysis. Underlying data is presented in Supplement 3, http://links.Iww.com/SLA/B435. * Adjusted for: propensity score, age, sex, BMI, ASA, Charlson comorbidity index, ECOG, tumor location, preoperative organ involvement, multivisceral resection, porto-mesenteric vein resection and histological diagnosis. ASA indicates American Society of Anesthesiologists; BMI, body mass index; C, center; ECOG, Eastern Cooperative Oncology Group; Excl., excluding; MV, multivariable; p, per; y, year.

$10 \%$ higher rate of grade $\mathrm{B} / \mathrm{C} \mathrm{POPF}$ and longer (90 minutes) operative times, but no increase in bile leakage. These results remained similar after excluding, respectively, centers performing 10 to 20 MIPDs per year, the first 20 MIPD procedures per center, hybrid procedures, conversions, and hybrid and laparoscopic procedures. No differences in risk of major morbidity, mortality, and POPF were observed between laparoscopic, robot-assisted, and hybrid MIPD, but the conversion rate was lower after robot-assisted- versus laparoscopic MIPD (5\% vs 26\%). Single-row pancreatojejunostomy was a newly identified risk factor for POPF in MIPD.

In keeping with these findings, 2 recent systematic reviews comparing MIPD versus OPD found no difference in 30-day mortality, but increased operative times after MIPD. ${ }^{1,8}$ In contrast to our findings, these studies found similar rates of POPF, lower rates of delayed gastric emptying, and shorter hospital stays after MIPD. One review also found fewer postoperative complications and a lower rate of delayed gastric emptying after robot-assisted- versus laparoscopic MIPD. As was noted in one of the reviews, considerable publication bias has likely influenced these positive results for MIPD. ${ }^{1}$ Two US nationwide registry studies on MIPD versus OPD for pancreatic ductal adenocarcinoma found higher rates of 30-day mortality in centers performing a fewer than 10 MIPDs annually, compared with equivalent rates in higher volume centers. ${ }^{15,16}$ Two other registry studies on MIPD versus OPD found that laparoscopic MIPD was associated with lower rates of overall complications and shorter hospital stays. ${ }^{14,18}$ Shorter hospital stays were also reported by the recent PLOT trial including 64 patients. ${ }^{19}$ The absence of a clinical benefit of MIPD in the current study is an important finding. However, given the current early experience and relatively low annual volume of MIPD in most participating centers it may be too soon for definitive conclusions on the merits of MIPD.

The $10 \%$ absolute increase of POPF after MIPD warrants further inquiry as this has been reported in small single center cohort studies, ${ }^{34,35}$ but not in large registry studies ${ }^{14-16,18}$ or systematic reviews. ${ }^{1,5,8}$ Because no differences in radiologic drainage or reoperations were observed between groups, the higher POPF rate is likely the result of prolonged drainage (ie, leaving the surgical drain in situ). Besides the approach itself, 2 other factors could have influenced this outcome; insufficient balance between groups at baseline (residual confounding) or underreporting of POPF in the German and Dutch registry data. First, residual confounding seems less likely, as OPD control patients were retrieved from unselected populations, with less than 5\% MIPD use, and important risk factors associated with POPF (BMI, pancreatic texture, vascular involvement $)^{36,37}$ were controlled for. Although pancreatic duct size was often missing, the result of a secondary matched analysis on patients with available duct size was not different from the primary analysis. Second, although the rates of grade B/C POPF in the German and Dutch data on OPD were similar $(11 \%)$, the 2005 ISGPS definition ${ }^{26}$ can be interpreted in different ways $^{38}$ and POPF may not always be accurately scored postdischarge in registry databases. Indeed, recent large retrospective single-center studies on OPD have reported grade B/C POPF rates between $17 \%^{5,39}$ and $28 \%{ }^{40}$ and prospective studies have reported rates between $17 \%{ }^{41}$ and $25 \% .^{42}$ These proportions are closer to the $23 \%$ POPF rate after MIPD found by this study. Moreover, the Indian PLOT trial found no increase in grade B/C POPF between laparoscopic MIPD and OPD $(6 \%$ vs $13 \%, P=0.311)$ with an annual volume over 40 pancreatectomy procedures and a total experience of over 150 MIPDs. ${ }^{19}$ Ultimately, more (multicenter) randomized trials are needed to compare POPF rates between MIPD and OPD.

This study has some limitations. First, we collected OPD controls from validated nationwide surgical registries with $<5 \%$ MIPD implementation and applied propensity score matching to minimize confounding by indication (surgical case selection). However, this type of bias can only be maximally avoided by randomization. Second, because MIPD data were retrieved from 
institutional databases and OPD data was retrieved from nationwide registries, differences in data collection and follow-up methods may have introduced information bias. Because major morbidity (requiring reinterventions), mortality, and length of stay are hard outcomes, this is less of a concern for the main study conclusions. Third, we could not compare levels of estimated blood loss or grade A POPF (ie, biochemical leak), because these variables were not recorded in the nationwide registries. Blood loss, however, is not an essential risk factor in the recent alternative fistula risk score (aFRS). ${ }^{36}$ Fourth, a variety of centers with different settings (academic vs nonacademic) and geographical characteristics were included. The result is substantial heterogeneity in preoperative workup, treatment strategies, and postoperative management between centers. Although this improves the external validity of the study, it reduces the accuracy of measuring the association between the approach and outcomes.

This is the first international multicenter matched study on MIPD versus OPD to date. A predefined study protocol was registered at clinicaltrials.gov to improve the study's validity and centers were offered anonymity to reduce reporting bias. The results give valuable insights into the outcomes of MIPD in the early experience of European centers performing at least 10 MIPDs per year. Consequently, our results only apply to centers with similar characteristics.

Moving forward, there are 2 major determinants of successful dissemination of MIPD: (i) the use of dedicated training programs and (ii) total- and annual procedure-specific case volumes. First, as previously shown in the Netherlands for laparoscopic distal pancreatectomy (LAELAPS-1 ${ }^{43}$ and laparoscopic pancreatoduodenectomy (LAELAPS-2), ${ }^{44}$ nationwide training programs can result in safe implementation of minimally invasive pancreatic surgery. In addition, a reproducible Institutional Training and Fellowship program for robot-assisted pancreatoduodenectomy, like the Pittsburgh example, has been shown to produce excellent outcomes. ${ }^{10,45}$ For example, their grade B/C POPF rate improved from $27.5 \%$ to $14.4 \%(P=$ $0.04)$ after the first 40 MIPDs. The LAELAPS-3 program was based on the Pittsburgh program and is currently being used, including Pittsburgh-based proctors, to implement robot-assisted MIPD within the Dutch Pancreatic Cancer Group. ${ }^{46}$ Second, 2 recent US nationwide studies found a volume-outcome associations for MIPD and defined a minimum volume cut-off of 22 to 25 MIPDs per year to achieve comparable outcomes to OPD. ${ }^{47,48}$ In the current study, no clear association between volume and POPF was found, but it should be noted that only 6 of 14 centers performed more than 20 MIPDs per year and only one center performed more than 40 MIPDs per year. Future studies with more centers reaching higher annual casevolumes are therefore needed.

This study found that MIPD is associated with similar 30-day major morbidity, mortality, and length of stay, but longer operative times and a $10 \%$ higher rate of grade B/C POPF compared with OPD. No differences in outcomes were found between robot-assisted-, laparoscopic- and hybrid MIPD, with lower conversion rates after robotassisted MIPD, although this sub group analysis was not the primary aim of the current study. In contrast to prior expectations, this study did not find the secondary benefits (eg, shorter hospital stays) to support the immediate widespread implementation of MIPD. We conclude that MIPD does not increase major morbidity or 30-day mortality in centers performing at least 10 MIPDs per year. However, the impact of anastomotic technique, higher annual case-volumes, and experience on outcomes (eg, grade B/C POPF) should be addressed in (prospective) studies before the true impact of MIPD can be established.

\section{ACKNOWLEDGMENTS}

The authors would like to acknowledge the office of the European-African Hepato-Pancreato-Biliary Association for supporting this study.
E-MIPS study group members: The authors are grateful for the contributions made by Thijs de Rooij (AMC, Amsterdam), Edward Willems (AZG, Kortrijk), Régis Fara (Hôpital Européen Marseille), Patrick Pessaux (Institut Hospitalo-Universitaire de Strasbourg), Bergthor Björnsson (Linköping University), Adelmo Antonucci (Policlinico di Monza), and Stefano Berti (S. Andrea Hospital, La Spezia).

\section{REFERENCES}

1. De Rooij T, Lu MZ, Steen MW, et al. Minimally invasive versus open pancreatoduodenectomy: systematic review and meta-analysis of comparative cohort and registry studies. Ann Surg. 2016;264:257-267.

2. Langan RC, Graham JA, Chin AB, et al. Laparoscopic-assisted versus open pancreaticoduodenectomy: early favorable physical quality-of-life measures. Surgery. 2014;156:379-384.

3. Birkmeyer JD, Stukel TA, Siewers AE, et al. Surgeon volume and operative mortality in the United States. N Engl J Med. 2003;349:2117-2127.

4. Orti-Rodriguez RJ, Rahman SH. A comparative review between laparoscopic and robotic pancreaticoduodenectomies. Surg Laparosc Endosc Percutan Tech. 2014;24:103-108.

5. Correa-Gallego C, Dinkelspiel HE, Sulimanoff I, et al. Minimally-invasive vs open pancreaticoduodenectomy: Systematic review and meta-analysis. J Am Coll Surg. 2014;218:129-139.

6. Edwin B, Sahakyan MA, Abu Hilal M, et al. Laparoscopic surgery for pancreatic neoplasms: the European association for endoscopic surgery clinical consensus conference. Surg Endosc. 2017;31:2023-2041.

7. De Rooij T, Klompmaker S, Abu Hilal M, et al. Laparoscopic pancreatic surgery for benign and malignant disease. Nat Rev Gastroenterol Hepatol. 2016;13:227-238.

8. Ricci C, Casadei R, Taffurelli G, et al. Minimally invasive pancreaticoduodenectomy: what is the best "choice"? A systematic review and network metaanalysis of non-randomized comparative studies. World J Surg. 2018;42:1-18.

9. Kendrick ML, Cusati D. Total laparoscopic pancreaticoduodenectomy: feasibility and outcome in an early experience. Arch Surg. 2010;145:19-23.

10. Boone BA, Zenati M, Hogg ME, et al. Assessment of quality outcomes for robotic pancreaticoduodenectomy: identification of the learning curve. JAMA Surg. 2015;150:416-422.

11. Palanivelu C, Rajan PS, Rangarajan M, et al. Evolution in techniques of laparoscopic pancreaticoduodenectomy: a decade long experience from a tertiary center. J Hepatobiliary Pancreat Surg. 2009;16:731-740.

12. Kim SCC, Song KBB, Jung YSS, et al. Short-term clinical outcomes for 100 consecutive cases of laparoscopic pylorus-preserving pancreatoduodenectomy: improvement with surgical experience. Surg Endosc. 2013;27: 95-103.

13. Klompmaker S, van Zoggel D, Watkins AAA, et al. Nationwide evaluation of patient selection for minimally invasive distal pancreatectomy using American College of Surgeons' National Quality Improvement Program. Ann Surg. 2017;266:1055-1061.

14. Zimmerman AM, Roye DG, Charpentier KP. A comparison of outcomes between open, laparoscopic and robotic pancreaticoduodenectomy. $H P B$ (Oxford). 2018;20:364-369.

15. Adam MA, Choudhury K, Dinan MA, et al. Minimally invasive versus open pancreaticoduodenectomy for cancer: practice patterns and short-term outcomes among 7061 patients. Ann Surg. 2015;262:372-377.

16. Sharpe SM, Talamonti MS, Wang CE, et al. Early national experience with laparoscopic pancreaticoduodenectomy for ductal adenocarcinoma: a comparison of laparoscopic pancreaticoduodenectomy and open pancreaticoduodenectomy from the National Cancer Data Base. J Am Coll Surg. 2015; 221:175-184.

17. van der Geest LG, van Rijssen LB, Molenaar IQ, et al. Volume-outcome relationships in pancreatoduodenectomy for cancer. HPB (Oxford). 2016;18:317-324.

18. Tran TB, Dua MM, Worhunsky DJ, et al. The first decade of laparoscopic pancreaticoduodenectomy in the United States: costs and outcomes using the nationwide inpatient sample. Surg Endosc. 2016;30:1778-1783.

19. Palanivelu C, Senthilnathan P, Sabnis SC, et al. Randomized clinical trial of laparoscopic versus open pancreatoduodenectomy for periampullary tumours. Br J Surg. 2017;104:1443-1450.

20. Von Elm E, Altman DG, Egger M, et al. The strengthening the reporting of observational studies in epidemiology (STROBE) statement: guidelines for reporting observational studies. Ann Intern Med. 2007;147:573-577.

21. Wellner UF, Klinger C, Lehmann $\mathrm{K}$, et al. The pancreatic surgery registry (StuDoQ|Pancreas) of the German Society for General and Visceral 
Surgery (DGAV): presentation and systematic quality evaluation. Trials. 2017;18:163.

22. van Rijssen LB, Koerkamp BG, Zwart MJ, et al. Nationwide prospective audit of pancreatic surgery: design, accuracy, and outcomes of the Dutch Pancreatic Cancer Audit. HPB (Oxford). 2017;19:919-926.

23. Dindo D, Demartines N, Clavien PA. Classification of surgical complications: a new proposal with evaluation in a cohort of 6336 patients and results of a survey. Ann Surg. 2004;240:205-213.

24. Charlson ME, Pompei P, Ales KL, et al. A new method of classifying prognostic comorbidity in longitudinal studies: development and validation. J Chron Dis. 1987;40:373-383.

25. Ament R. Origin of the ASA classification. Anesthesiology. 1979;51:179.

26. Bassi C, Dervenis C, Butturini G, et al. Postoperative pancreatic fistula: an international study group (ISGPF) definition. Surgery. 2005;138:8-13.

27. Bassi C, Marchegiani G, Dervenis C, et al. The 2016 update of the International Study Group (ISGPS) definition and grading of postoperative pancreatic fistula: 11 years after. Surgery. 2017;161:584-591.

28. Wente MN, Bassi C, Dervenis C, et al. Delayed gastric emptying (DGE) after pancreatic surgery: a suggested definition by the International Study Group of Pancreatic Surgery (ISGPS). Surgery. 2007;142:761-768.

29. Wente MN, Veit JA, Bassi C, et al. Postpancreatectomy hemorrhage (PPH): an International Study Group of Pancreatic Surgery (ISGPS) definition. Surgery. 2007;142:20-25.

30. Koch M, Garden OJ, Padbury R, et al. Bile leakage after hepatobiliary and pancreatic surgery: a definition and grading of severity by the International Study Group of Liver Surgery. Surgery. 2011;149:680-688.

31. Mangram AJ, Horan TC, Pearson ML, et al. Guideline for Prevention of Surgical Site Infection, 1999. Centers for Disease Control and Prevention (CDC) Hospital Infection Control Practices Advisory Committee. Am J Infect Control. 1999;27:97-132.

32. Lonjon G, Porcher R, Ergina P, et al. Potential pitfalls of reporting and bias in observational studies with propensity score analysis assessing a surgical procedure: a methodological systematic review. Ann Surg. 2017;265:901-909.

33. White IR, Royston P, Wood AM. Multiple imputation using chained equations: Issues and guidance for practice. Stat Med. 2011;30:377-399.

34. Boggi U, Napoli N, Costa F, et al. Robotic-assisted pancreatic resections. World J Surg. 2016;40:2497-2506.

35. Lai EC, Yang GP, Tang CN. Robot-assisted laparoscopic pancreaticoduodenectomy versus open pancreaticoduodenectomy: a comparative study. Int J Surg. 2012;10:475-479.
36. Mungroop TH, van Rijssen LB, van Klaveren D, et al. Alternative fistula risk score for pancreatoduodenectomy (a-FRS): design and international external validation. Ann Surg. 2017 [Epub ahead of print].

37. Callery MP, Pratt WB, Kent TS, et al. A prospectively validated clinical risk score accurately predicts pancreatic fistula after pancreatoduodenectomy. $J$ Am Coll Surg. 2013;216:1-14.

38. Pulvirenti A, Ramera M, Bassi C. Modifications in the International Study Group for Pancreatic Surgery (ISGPS) definition of postoperative pancreatic fistula. Transl Gastroenterol Hepatol. 2017;2:107.

39. Correa-Gallego C, Brennan MF, D'Angelica M, et al. Operative drainage following pancreatic resection: analysis of 1122 patients resected over 5 years at a single institution. Ann Surg. 2013;258:1051-1058.

40. Pulvirenti A, Marchegiani G, Pea A, et al. Clinical implications of the 2016 International Study Group on pancreatic surgery definition and grading of postoperative pancreatic fistula on 775 consecutive pancreatic resections. Ann Surg. 2017 [Epub ahead of print].

41. Kawai M, Tani M, Terasawa $H$, et al. Early removal of prophylactic drains reduces the risk of intra-abdominal infections in patients with pancreatic head resection: prospective study for 104 consecutive patients. Ann Surg. 2006;244:1-7.

42. Bassi C, Molinari E, Malleo G, et al. Early versus late drain removal after standard pancreatic resections: results of a prospective randomized trial. Ann Surg. 2010;252:207-214.

43. de Rooij T, van Hilst J, Boerma D, et al. Impact of a nationwide training program in minimally invasive distal pancreatectomy (LAELAPS). Ann Surg. 2016;264:754-762.

44. de Rooij T, van Hilst J, Topal B, et al. Outcomes of a multicenter training program in laparoscopic pancreatoduodenectomy (LAELAPS-2). Ann Surg. 2017 [Epub ahead of print].

45. Hogg ME, Tam V, Zenati M, et al. Mastery-based virtual reality robotic simulation curriculum: the first step toward operative robotic proficiency. $J$ Surg Educ. 2017;74:477-485.

46. Nota CL, Zwart MJ, Fong Y, et al. Developing a robotic pancreas program: the Dutch experience. J Vis Surg. 2017;3:106.

47. Adam MA, Thomas S, Youngwirth L, et al. Defining a hospital volume threshold for minimally invasive pancreaticoduodenectomy in the United States. JAMA Surg. 2017;152:336-342.

48. Kutlu OC, Lee JE, Katz MH, et al. Open pancreaticoduodenectomy case volume predicts outcome of laparoscopic approach: a population-based analysis. Ann Surg. 2018;267:552-560. 